\title{
Selection for altruistic defense in structured populations
}

\author{
Felix Jordan ${ }^{1}$, Martin Hutzenthaler ${ }^{2}$, and Dirk Metzler*1 \\ ${ }^{1}$ Division of Evolutionary Biology, Faculty of Biology, LMU Munich, \\ Großhaderner Str. 2, 82152 Martinsried, Germany, \\ felix.jordan@web.de/metzler@bio.lmu.de \\ ${ }^{2}$ Dept. of Mathematics, University of Duisburg-Essen, \\ Thea-Leymann-Str. 9, 45127 Essen, Germany, \\ martin.hutzenthalereuni-due.de
}

August 12, 2019

Research supported by the DFG in the Priority Program "Probabilistc Structures in Evolution" (SPP 1590), grants HU 1889/3-2 and ME 3134/6-2.

\begin{abstract}
We model natural selection for or against an altruistic defense allele of a host (or prey) against a parasite (or predator). The populations are structured in demes and we specify rates for birth, death, and migration events of single individuals. The defense behavior has a fitness cost for the actor and locally reduces parasite growth rates. In a previous study (Hutzenthaler et al., 2015), we analytically derived a criterion for fixation or extinction of altruists in the limit of large populations, many demes, weak selection and slow migration. Here, we use two simulation approaches to analyze the model in relaxed settings. We confirm that the criterion still holds for settings with finitely many demes with various migration patterns if populations are large and the ecological interactions are fast compared to evolutionary processes. For smaller populations with no complete separation of evolutionary and ecological time scales, the value of the shift between fixation and extinction changes, but the qualitative insights remain valid. The key mechanism of providing a benefit of altruism is randomness of reproduction and death events leading to differences in population sizes between demes. Randomness, which is more pronounced for small populations, improves the conditions for fixation of the altruistic allele. Furthermore, as suggested by the previous asymptotic results, we find no significant effect of the migration rate and conclude that the amount of gene flow under which the evolution of altruism is favored may not be as limited as suggested by previous studies.
\end{abstract}

*corresponding author 


\section{Introduction}

Any trait or character that harms the reproductive success of its bearer while simultaneously increasing the reproductive success of other individuals is called altruistic (e.g., West et al., 2007). The evolutionary success of such traits seems unlikely when considering the individual as the unit of selection (Hamilton, 1963). Nevertheless, numerous examples of altruism are discussed in the literature, such as sterility in insects (e.g., Hölldobler and Wilson, 2009), food sharing in vampire bats (Wilkinson, 1984, 1990), and production of costly public goods in bacteria (Diggle et al., 2007; Williams et al., 2007). Explanations of how an altruistic trait can evolve are provided by the theories of kin selection (Hamilton, 1964a,b) and group selection (Wynne-Edwards, 1963; Queller, 1992). Also evolutionary game theory has provided important insights into the evolution of altruism and cooperation (Axelrod and Hamilton, 1981; Nowak and May, 1992). The apparent disadvantage of altruism may be overcome if spatial structure or repeated interactions are considered (Nowak, 2006; Ohtsuki et al., 2006).

The theory of kin selection (also known as inclusive fitness theory) provides the insight that not only the focal individual should be considered when evaluating the success of a trait, but also the effects on its interaction partners. The most prominent result of kin selection theory is Hamilton's rule, stating that an altruistic act is selectively favored if the benefit to others, weighted by the relatedness of the actor to the beneficiaries, is greater than the cost to the actor (Hamilton, 1964a,b).

An alternative approach to explain the evolution of altruism and cooperation is group selection or multi-level selection theory (see Traulsen and Nowak, 2006; Gardner, 2015, for an overview). When individuals live in distinct groups that are in competition with each other, an advantage on the group level may under certain conditions outweigh a disadvantage on the level of individuals (Wynne-Edwards, 1963; Maynard Smith, 1964; Uyenoyama, 1979; Wade, 1982; Bijma et al., 2007). According to the equation of Price (1970), the selection pressure on a trait is the covariance between the trait and its fitness effects. For the case of subdivided populations Queller (1992) separated the within- and between group components of this covariance in Price's equation. Even if the covariance of a trait with its fitness is negative within each group, it can still be positive in the whole population. This can be understood as an instance of what is known in statistics as the Yule-Simpson effect (Yule, 1903; Simpson, 1951; Blyth, 1972; Sober and Wilson, 1998; Gardner, 2015; Metzler et al., 2016). A necessary condition for this effect is, however, that frequencies of the trait vary between the groups, and an interesting question is what factors besides the kinship structure of the population could maintain this variance over evolutionarily relevant time spans. The theories of kin and group selection have been intensely debated (Traulsen and Nowak, 2006; Nowak et al., 2010; Abbot et al., 2011; Rousset and Lion, 2011; Van Veelen et al., 2012; Gardner, 2015). Birch and Okasha (2015) argue that some of the controversies about inclusive fitness theory and its relationship to group selection theory result from disparate interpretations of the terms in Hamilton's rule.

Traits of defense against parasites (or predators) can be altruistic as they may be costly for the carrier of the trait while reducing its neighbors' risk of being infected (or attacked). Costly defense traits are known for many species (Siva-Jothy et al., 2005), including, e.g., resistance against virus infection in meal moths and antibacterial ac- 
tivity in cotton leaf worms (Cotter et al., 2004). Antonovics and Thrall (1994) and Bowers et al. (1994) modeled the dynamics of host-parasite systems for the case that there is an inheritable trait in the host species that makes its carriers less susceptible but also reduces their reproduction rate (see Boots et al., 2009, for a review of extensions of these models). There is, however, no spatial structure in these models and thus no kin or group selection in favor of this resistance trait. Models for the evolution of defense traits in spatially structured host populations have been analyzed by Frank (1998), Brown and Hastings (2003), Schliekelman (2007), Best et al. (2011) and Débarre et al. (2012). Pamminger et al. (2014) analyzed the population structure of ant populations that are the host of a social parasite, a "slavemaking" ant species, and concluded that a defense trait of "slave rebellion" could evolve via kin selection as it reduces the parasite pressure on neighboring ant nests that are likely to be closely related to the "rebels". Metzler et al. (2016) carried out extensive computer simulation studies for the system of Pamminger et al. (2014) and found that the evolution of slave rebellion may in principle be possible, but only for a narrow range of parameter combinations leading to a meta-population dynamic with kin/group selection on a larger spatiotemporal scale than considered by Pamminger et al. (2014).

When altruism takes the form of defense against a parasite (or predator), the success of the costly defense trait depends on the presence of the parasite. Once altruists become abundant, they reduce the number of parasites, which in turn may reduce the benefit that each altruist provides. It is not a priori clear how this feedback mechanism affects the evolution of the defense trait under kin or group selection. It may for example lead to diminishing returns of the amount of altruism, i.e., the more altruists are present, the lower the additional benefit of even more altruists. Sibly and Curnow (2011) show that coexistence of altruism and cheating will occur if cooperation is subject to diminishing returns. Furthermore, the benefits of defense are mediated by the parasite population, such that there may be a time lag between an altruistic act and the increase in reproductive success of host individuals.

In Hutzenthaler et al. (2015) we have analytically investigated the scenario of altruistic defense in structured populations. For this we used a model of diffusion equations for the frequencies of altruists, cheaters, and parasites and analyzed the asymptotic properties of the model in the limit of many demes of large subpopulations with little migration between the demes and weak selection. We found that altruists will go to fixation if $\alpha$, the selective disadvantage of altruist compared to cheaters in the same deme, is greater than $\beta$, the effect of altruistic defense scaled by the relative strength of parasites and the amount of randomness in host reproduction. Conversely, altruism will go extinct if $\alpha<\beta$. Coexistence of altruists and cheaters occurs only in the critical case $\alpha=\beta$. An overview of the derivation in Hutzenthaler et al. (2015) is shown in Figure 1.

Here, we simulate host-parasite (or predator-prey) interactions according to an individual-based model in which the populations evolve via single birth, death, and migration events of single individuals - one event at a time. The model does not rely on specific mechanisms to provide an advantage of altruism (such as extinction or proliferation of whole demes) and no relatedness values need to be computed. Hutzenthaler et al. (2015) showed for the limit of this model for large populations and many demes with an infinite time horizon that there is a strict separation between parameter 


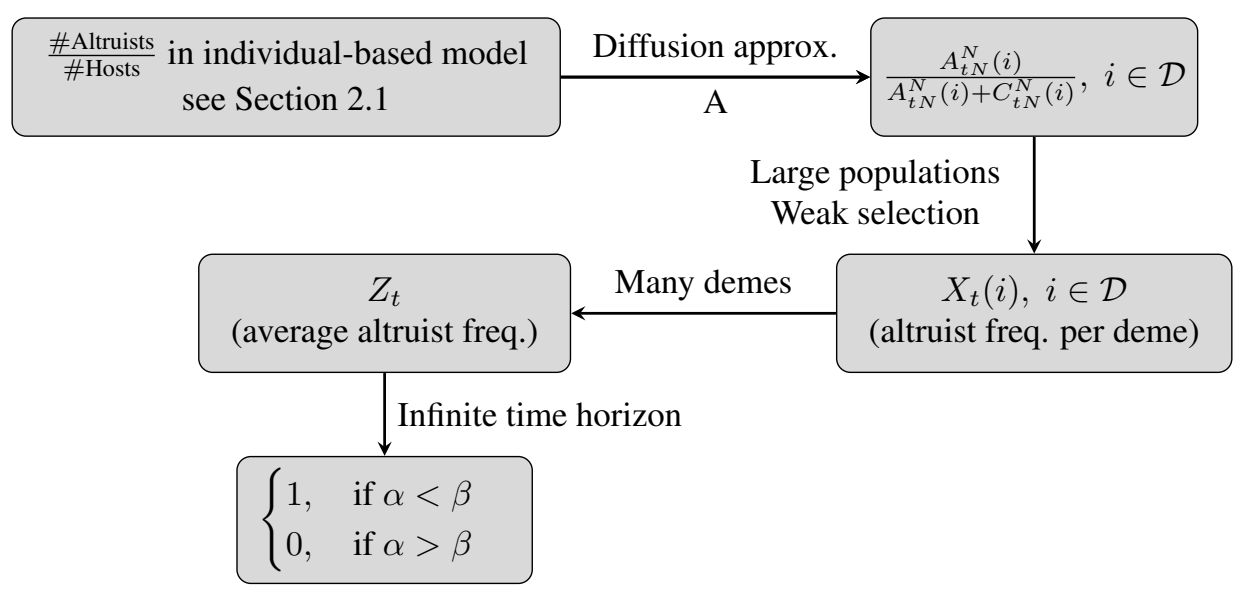

Figure 1: Overview of the results from the theoretical analysis on the relative frequency of an altruistic defense allele in a structured host population. Here, $\mathcal{D}$ denotes the set of demes. The individual-based model forming the basis of the analysis is defined in Section 2.1. A provides the diffusion approximation leading to the diffusion model that is the starting point of Hutzenthaler et al. (2015). (Therein, it is mentioned, that the model can be derived from an individiual-based ("particle") model. A closes this gap.) Assuming weak selection (i.e., a weak reproductive cost of altruistic defense) and weak migration, in the limit of infinite population sizes a simplified process $X$ describing the relative altruist frequency per deme on the evolutionary time scale is derived. In the limit of infinitely many demes with uniform migration, the mean-field limit $Z$ of this process is obtained, describing the average altruist frequency per deme. Finally, analyzing the long-term behavior of this average frequency, the condition for fixation or extinction of altruists is determined (see Section 1 for details). In the notation of Table 1, we have $\beta=\beta_{H} \rho \frac{\delta}{\delta \nu+\lambda \gamma}$.

combinations that lead to fixation or extinction of altruism. Here we investigate the relevance of these results in the finite scenario of the individual-based model as well as the setting of large populations living in finitely many demes. Note that the models analyzed here and in Hutzenthaler et al. (2015) do not include the possibility that hosts become immune by contact with parasites. Therefore, our model may not be applicable to host-parasite systems in which immunization is crucial but are, on the other hand, very generic and therefore also applicable to predator-prey systems.

In Section 2.1 we will first introduce the individual-based model that we use for computer simulations with finite population sizes and finitely many demes to assess the validity of the prediction from the idealized settings in Hutzenthaler et al. (2015). We evaluate the influences of randomness in reproduction, intensity of gene flow, and fluctuations in competition strengths on the success of altruism. Furthermore, we simulate the altruist frequency under infinite population sizes and a complete separation of time scales (process $X$ in Figure 1) to investigate the effect of these assumptions. Finally, we simulate different migration patterns to assess their influence on the success 
of altruism.

\section{Methods}

\subsection{Individual-based simulation model for small population sizes}

We will now define an individual-based model for the evolution of an altruistic defense trait. The model of Hutzenthaler et al. (2015) can be derived as a diffusion approximation of a special case of this model; see A for details. The states of our model are tuples of numbers of altruists, cheaters (i.e., non-altruistic host individuals), and parasites in $D$ demes. For each of these types of individuals, birth, death, and migration rates are specified. These rates incorporate randomness in reproduction, competition within species (to account for limited space and resources per deme), and interactions between hosts and parasites. Hosts suffer from parasite presence, while parasites need hosts to survive and grow. Altruists provide the benefit of locally reducing parasite growth and pay the cost of a reduced reproduction rate compared to cheaters. All parameters of the model and their values in our simulation study are shown in Table 1 and the resulting transition rates in Box 1. We interprete the time unit as one generation, but the parameters $\beta_{H}$ and $\beta_{P}$ allow to bring additional randomness into the model to cover, e.g., effects of skewed offspring distributions or other factors that could affect the effective population size and thus genetic drift in the host. The chance of the defense trait to go to fixation depends on $\beta=\beta_{H} \rho \frac{\delta}{\delta \nu+\lambda \gamma}$, where $\rho$ denotes the decrease in parasite growth rate per altruist, $\delta$ is the host death rate per parasite, $\nu$ denotes the parasite death rate, $\lambda$ defines the host birth rate, $\gamma$ is the competition rate among parasites, and $\beta_{H}$ describes the randomness in host reproduction (Hutzenthaler et al., 2015).

The initial sizes of altruists, cheaters, and parasites in each deme are $A_{0} N, C_{0} N$, and $P_{0} N$. In each simulation step, a single transition is performed, which can be a birth, death, or migration event for one individual of one of the three types in any deme. The inverse of the sum of all transition rates is added to the time that has passed. This corresponds to the expected value of the time until the next transition occurs, which is used instead of the random value for computational efficiency. (The random value is exponentially distributed with a rate of the sum of all transition rates.) The transition is randomly chosen with probabilities proportional to the transition rates, which are given in Box 1. After the numbers of individuals are updated with respect to the randomly chosen transition event, the transition rates are adjusted accordingly. If the time that has passed exceeds the specified time horizon $T$ or if altruists or cheaters go to fixation, then the simulation run terminates. Otherwise, the next transition step is performed.

\subsubsection{Simulation study}

In simulation series $\alpha$, we simulated the individual-based model with different values of altruist disadvantage $\alpha$ and different values of the effect of altruistic defense, $\rho$, which led to different values of the $b=\rho \frac{\delta}{\delta \nu+\lambda \gamma}=\beta / \beta_{H}$, which is a measure for the benefit of defense for the local host population (Hutzenthaler et al., 2015). To further understand how the randomness in the finite model affects the outcome, simu- 


\begin{tabular}{|c|c|c|c|c|c|}
\hline Par. & Description & $\boldsymbol{\alpha}$ & $\boldsymbol{\beta}_{\boldsymbol{H}}$ & $\boldsymbol{\kappa}_{\boldsymbol{H}}$ & $\boldsymbol{a}_{\boldsymbol{f}}$ \\
\hline$N$ & population size scaling & 500 & 500 & 500 & 500 \\
\hline$D$ & number of demes & 50 & 50 & 50 & 50 \\
\hline$T$ & time horizon & $2.5 \cdot 10^{6}$ & $2.5 \cdot 10^{6}$ & $2.5 \cdot 10^{6}$ & $2.5 \cdot 10^{6}$ \\
\hline$A_{0}$ & initial altruists per deme (units of $N)$ & 1 & 1 & 1 & 1 \\
\hline$C_{0}$ & initial cheaters per deme (units of $N)$ & 1 & 1 & 1 & 1 \\
\hline$P_{0}$ & initial parasites per deme (units of $N)$ & 1 & 1 & 1 & 1 \\
\hline$\lambda$ & host growth rate & 1 & 1 & 1 & 1.5 \\
\hline$K$ & host carrying capacity (units of $N)$ & 2 & 2 & 2 & 2 \\
\hline$\delta$ & host death per parasite & 1 & 1 & 1 & 0.5 \\
\hline$\alpha$ & additional death rate of altruists & var & 1 & 1 & 1 \\
\hline$\nu$ & parasite death rate & 0.5 & 0.5 & 0.5 & 0.5 \\
\hline$\rho$ & parasite death per altruist & var & var & var & var \\
\hline$\gamma$ & parasite competition & 2 & 2 & 2 & 1.5 \\
\hline$\eta$ & parasite growth per host & 1 & 2 & 2 & 4 \\
\hline$\kappa_{H}$ & host migration rate & 1 & 1 & var & 1 \\
\hline$\kappa_{P}$ & parasite migration rate & 1 & 1 & 1 & 1 \\
\hline$\beta_{H}$ & randomness in host reproduction & 1 & var & 1 & 1 \\
\hline$\beta_{P}$ & randomness in parasite reproduction & 1 & 1 & 1 & 1 \\
\hline$p_{f}$ & probability of fluctuating competition & 0 & 0 & 0 & 0.02 \\
\hline$a_{f}$ & amount of competition fluctuation & 0 & 0 & 0 & var \\
\hline
\end{tabular}

Table 1: Parameters of individual-based model with values used in the different simulation series. Entries marked with "var" vary for the simulation series and the values are provided in the corresponding plots.

lation series $\beta_{H}$ was performed for various values of $\beta_{H}$. For finite population sizes $N<\infty$, the time scales of evolutionary and ecological forces are not separated and the fixation probability of the defense trait may depend on the migration rate. A low value of $\kappa_{H}$ means that effects within demes dominate the dynamics, while a very large value removes the effect of population structure. We investigated the influence of $\kappa_{H}$ in simulation series $\kappa_{H}$. In simulation series $a_{f}$, we relaxed the assumption of having identical conditions in each deme. After each generation, the competition rate was allowed to change in each deme, independently for hosts and parasites, each with probability of $p_{f}$. If such a change occurred, a normally distributed random number $r$ with mean zero and standard deviation $a_{f} \lambda / K N$ for hosts or $a_{f} \gamma / N$ for parasites was drawn. The new competition rate was then set to $\lambda / K N+r$ in the case of hosts or $\gamma / N+r$ in the case of parasites. If the resulting value was non-positive, a new random number was drawn (until a positive value was obtained). The effect of these fluctuations was investigated for different values of $a_{f}$. The values for all parameters in the different simulation series are shown in Table 1 . All data was analyzed using $R(\mathrm{R}$ Core Team, 2015). Kruskal-Wallis tests were performed to check for significance using the function kruskal.test and p-values were adjusted for multiple testing with the Bonferroni-Holm correction via the function p.adjust. 
Box 1: Transition rates when the numbers of altruists, cheaters, and parasites are $a_{k}, c_{k}$, and $p_{k}$, respectively, in all demes $k \in \mathcal{D}$, with $\mathcal{D}:=\{1, \ldots, D\}$. For all $i, j \in \mathcal{D}$, the rates are given by

$$
\begin{array}{ll}
\left(a_{k}, c_{k}, p_{k}\right)_{k \in \mathcal{D}} \rightarrow\left(a_{k}+\mathbb{1}_{k=i}, c_{k}, p_{k}\right)_{k \in \mathcal{D}}: & a_{i}\left(\beta_{H}+\lambda\right) \\
\left(a_{k}, c_{k}, p_{k}\right)_{k \in \mathcal{D}} \rightarrow\left(a_{k}-\mathbb{1}_{k=i}, c_{k}, p_{k}\right)_{k \in \mathcal{D}}: & a_{i}\left(\beta_{H}+\frac{\lambda}{K} \frac{a_{i}+c_{i}}{N}+\delta \frac{p_{i}}{N}+\frac{\alpha}{N}\right) \\
\left(a_{k}, c_{k}, p_{k}\right)_{k \in \mathcal{D}} \rightarrow\left(a_{k}, c_{k}+\mathbb{1}_{k=i}, p_{k}\right)_{k \in \mathcal{D}}: & c_{i}\left(\beta_{H}+\lambda\right) \\
\left(a_{k}, c_{k}, p_{k}\right)_{k \in \mathcal{D}} \rightarrow\left(a_{k}, c_{k}-\mathbb{1}_{k=i}, p_{k}\right)_{k \in \mathcal{D}}: & c_{i}\left(\beta_{H}+\frac{\lambda}{K} \frac{a_{i}+c_{i}}{N}+\delta \frac{p_{i}}{N}\right) \\
\left(a_{k}, c_{k}, p_{k}\right)_{k \in \mathcal{D}} \rightarrow\left(a_{k}, c_{k}, p_{k}+\mathbb{1}_{k=i}\right)_{k \in \mathcal{D}}: & p_{i}\left(\beta_{P}+\eta \frac{c_{i}}{N}+(\eta-\rho) \frac{a_{i}}{N}\right) \\
\left(a_{k}, c_{k}, p_{k}\right)_{k \in \mathcal{D}} \rightarrow\left(a_{k}, c_{k}, p_{k}-\mathbb{1}_{k=i}\right)_{k \in \mathcal{D}}: \quad p_{i}\left(\beta_{P}+\nu+\gamma \frac{p_{i}}{N}\right) \\
\left(a_{k}, c_{k}, p_{k}\right)_{k \in \mathcal{D}} \rightarrow\left(a_{k}-\mathbb{1}_{k=i}+\mathbb{1}_{k=j}, c_{k}, p_{k}\right)_{k \in \mathcal{D}}: \quad a_{i} \frac{\kappa_{H}}{N} \frac{1}{D} \\
\left(a_{k}, c_{k}, p_{k}\right)_{k \in \mathcal{D}} \rightarrow\left(a_{k}, c_{k}-\mathbb{1}_{k=i}+\mathbb{1}_{k=j}, p_{k}\right)_{k \in \mathcal{D}}: \quad c_{i} \frac{\kappa_{H}}{N} \frac{1}{D} \\
\left(a_{k}, c_{k}, p_{k}\right)_{k \in \mathcal{D}} \rightarrow\left(a_{k}, c_{k}, p_{k}-\mathbb{1}_{k=i}+\mathbb{1}_{k=j}\right)_{k \in \mathcal{D}}: \quad p_{i} \frac{\kappa_{P}}{N} \frac{1}{D}
\end{array}
$$

where $\mathbb{1}_{k=i}=1$, if $k=i$ and $\mathbb{1}_{k=i}=0$, otherwise. Thus, $\left(a_{k}+\mathbb{1}_{k=i}, c_{k}, p_{k}\right)_{k \in \mathcal{D}}$ refers to a birth event of altruists in deme $i$, i.e., the number of altruists in deme $i$ is increased by one, while no other value changes.

\subsection{Simulation model for altruist frequency in large populations}

In this section we introduce the simulation approach of the limit process $X$ for the altruist frequency in large populations on the evolutionary time scale (Figure 1). Thus, time is scaled in units of $N$ generations to capture the effects of weak selection and slow migration. The dynamics of $X$ is given by equation (9) in B and the approximation is done in the following way. At the beginning of the simulation, $X_{i}$ is set to $x_{0}$ in each deme $i \in\{1, \ldots, D\}$ and the current time $t$ is set to zero. The discretization is achieved by choosing a small, positive step size $d t$, and approximating the change of the Brownian motion in each time step by $d W_{i}$, for $i \in\{1, \ldots, D\}$, which is normally distributed with mean zero and standard deviation $\sqrt{d t}$. In each simulation step, corresponding to a time interval of length $d t$, the value of $X_{i}$ is updated as

$$
\begin{aligned}
X_{i}:=X_{i} & +\left[\kappa\left(a-X_{i}\right)\left(\sum_{j=1}^{D} m(i, j) \frac{a-X_{i}}{a-X_{j}}-1\right)-\alpha X_{i}\left(1-X_{i}\right)\right] d t \\
& +\sqrt{\beta\left(a-X_{i}\right) X_{i}\left(1-X_{i}\right)} d W_{i}
\end{aligned}
$$

for all $i \in\{1, \ldots, D\}$, where the migration rate $m(i, j)$ from deme $j$ to deme $i$ depends on the migration scheme that is used. Note that $\beta=\beta_{H} \rho \frac{\delta}{\delta \nu+\lambda \gamma}$ and $a=\frac{\lambda \gamma+\delta K \eta}{\delta K \rho}$ in the notation of Section 2.1. The parameter $a$ arises in the separation of time scales, when computing the equilibrium values of host and parasite population sizes for a given altruist frequency (see Hutzenthaler et al., 2015, for details). If $X_{i}<\varepsilon$, then $X_{i}$ is set to zero, or if $X_{i}>1-\varepsilon$, then $X_{i}$ is set to one (see C for details). Then, $t$ is 
Box 2: Migration patterns for the simulations series with uniform migration $(\mathrm{U})$, nearest-neighbor migration $(\mathrm{N})$, two-dimensional nearest-neighbor migration (2D), and migration along edges of a binary tree (T). Note that $m(i, j)$ refers to migration from deme $j$ to deme $i$.

$\mathbf{U} \quad m(i, j)=1 / D$ for all $i, j \in\{1, \ldots, D\}$

N $\quad m(i, j)=1 / 2$ if $|i-j|=1$ or $\{i, j\}=\{1, D\} ; m(i, j)=0$, otherwise

2D $m(i, j)=1 / 4$ if $i$ next to $j$ on two-dim. lattice; $m(i, j)=0$, otherwise

T $\quad m(i, j)=1 / 3$ if $j>1$ is parent or child of $i$ or if $i$ and $j$ are neighboring leaves; $m(i, j)=\frac{1}{2}$, if $j=1$ and $i \in\{2,3\} ; m(i, j)=0$, otherwise

increased by $d t$, and if $t$ is an integer-valued multiple of $T / 1000$, then the average value of $X$ across all demes is computed. If this average value is zero or one, or if $t \geq T$, then the simulation run is terminated.

\subsubsection{Simulation study}

To assess how well the results from the theoretical analysis could predict the outcome for finitely many demes, we performed simulations for a fixed value of altruist disadvantage $\alpha$ and different values of the benefit $\beta$. This was done for different values of the number of demes, $D$, to check how well the setting of infinitely many demes in the theoretical analysis could be approximated. In further analyses, the robustness of the system towards various migration schemes was investigated. The different schemes were uniform migration, nearest-neighbor migration on a circle, migration to the four immediate neighbors in a lattice on a two-dimensional torus, and migration to connected nodes on a binary tree, where the root was connected to its two children, any inner node was connected to its two children and its parent node, and any leaf was connected to its parent node as well as its two neighboring leaves (i.e., the leaves formed a circle along which migration occurs). See Box 2 for details on the migration patterns. The parameters used in the different settings are shown in Table 2. For each parameter set we performed 100 simulations.

\section{Results}

\subsection{Small populations}

In simulation series $\alpha, \beta_{H}, \kappa_{H}$, and $a_{f}$, the number of demes was set to $D=50$ and the initial numbers of altruists, cheaters and parasites per deme were $N=500$ each. For the values of the parameters see Table 1. For each parameter configuration, 100 simulation runs were performed. In simulation series $\alpha$ we tested the effect of finite population sizes on the theoretical prediction of Hutzenthaler et al. (2015) for different values of $\alpha$ and $b$ (where $b=\beta / \beta_{H}=\rho \delta / \delta \nu+\lambda \gamma$ in the notation of Section 2.1). The mean values of the results of the 100 simulations are shown in Figure 2a. All final 


\begin{tabular}{|c|c|c|c|c|c|}
\hline Par. & Description & $\mathbf{U}$ & $\mathbf{N}$ & $\mathbf{2 D}$ & $\mathbf{T}$ \\
\hline$\beta$ & random fluctuation (“benefit”) & var & var & var & var \\
\hline$\alpha$ & altruist disadvantage & 0.05 & 0.05 & 0.1 & 0.05 \\
\hline$\kappa$ & migration rate & 0.1 & 0.1 & 1 & 0.1 \\
\hline$a$ & see Section 2.2 & 2 & 2 & 2 & 3 \\
\hline$D$ & number of demes & var & var & var & var \\
\hline$x_{0}$ & initial altruist frequency per deme & 0.5 & 0.5 & 0.5 & 0.5 \\
\hline$T$ & time horizon & 2000 & 2000 & 2000 & 2000 \\
\hline$d t$ & time step size of discretization & $10^{-5}$ & $10^{-5}$ & $10^{-5}$ & $10^{-5}$ \\
\hline$\varepsilon$ & cutoff value & $10^{-7}$ & $10^{-7}$ & $10^{-7}$ & $10^{-7}$ \\
\hline
\end{tabular}

Table 2: Parameters of diffusion simulations with values for runs with uniform migration $(\mathrm{U})$, nearest-neighbor migration $(\mathrm{N})$, two-dimensional nearest-neighbor migration (2D), and migration along edges of a binary tree (T). Entries marked with "var" vary for the simulation series and the values are provided in the corresponding plots.

frequencies were either zero or one, i.e., extinction or fixation of altruists was observed in each run. The results do not exactly agree with the asymptotic that predicts extinction of altruists for $\alpha>b$ and fixation of altruists for $\alpha<b$ (note that $\beta_{H}=1$ is fixed in simulation series $\alpha$, such that $b=\beta$ ). Qualitatively, the trend can be seen, but $\alpha$ and $b$ have to be sufficiently far apart for fixation or extinction of altruists to be stable across different simulation runs with the same settings. For $\alpha=1$, extinction of altruists was observed in all 100 simulations for each of the values of $b$. For $\alpha=0.5$ and $\alpha=0.4$, there was no significant difference between the results for the different values of $b$ (Bonferroni-Holm corrected p-values of 0.37 and 0.10 , respectively). For all other values of $\alpha$, there was significant difference between the results for the different values of $b$ (all Bonferroni-Holm corrected p-values below 0.0002). Thus, for the selective disadvantage of altruists to be big enough, to overcome even small benefit effects of $b \leq 0.05, \alpha$ has to be sufficiently large.

Effects of randomness in host reproduction introduced by $\beta_{H}$ were investigated in simulation series $\beta_{H}$ (see Figure $2 b$ ). There is no significant difference between the results for the different values of $\beta_{H}$ for $b=0.6$ (Bonferroni-Holm corrected pvalue of 0.11 ). For all other values of $b$, there was a significant difference (maximum corrected p-value of $2 \cdot 10^{-6}$ ). In the simulations with $b=0.7$, the parasite population went extinct in $94,98,96$, and 82 runs out of the 100 runs for $\beta_{H}=0, \beta_{H}=0.1$, $\beta_{H}=1$, and $\beta_{H}=10$, respectively. For all other values of $b$, the parasite population never went extinct in any of the simulations.

In simulation series $\kappa_{H}$, we investigated the effect of host migration rates. Results are shown in Figure 2c. Among the simulations with $\kappa_{H}=0.01$, there were eight and five runs out of 100 total, for $b=0.2$ and $b=0.3$, respectively, where neither extinction nor fixation of altruists had occurred by the end of the simulation. This means there were both altruists and cheaters present in the host population and final altruist frequency was strictly between zero and one. For $b=0$ and $b=0.1$, all simulations led to extinction of altruists, while for $b=0.4$ and $b=0.5$, all simulations showed fixation of altruists. Intermediate values of $b$ showed no significant difference 
(a) simulation series $\alpha$

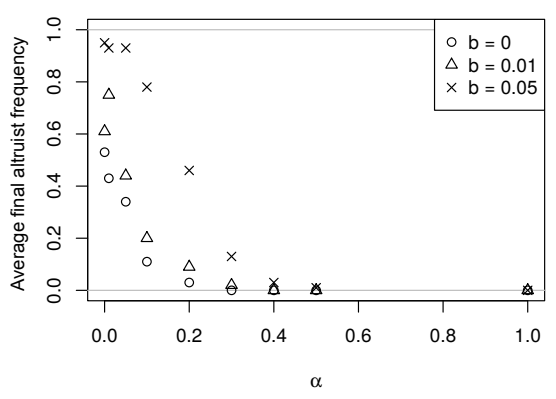

(c) simulation series $\kappa_{H}$

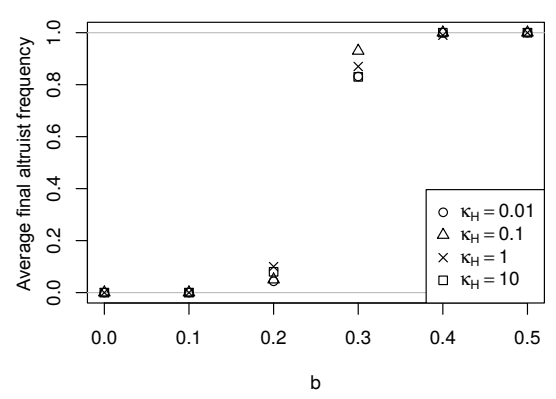

(b) simulation series $\beta_{H}$

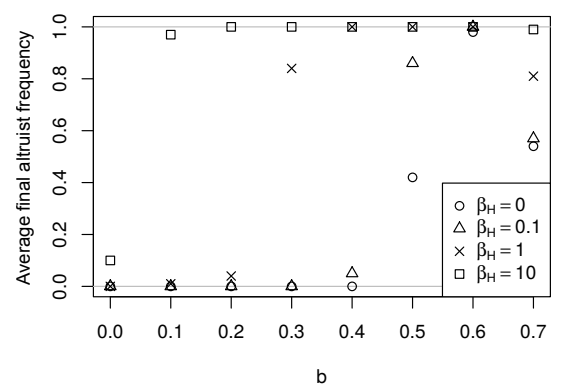

(d) simulation series $a_{f}$

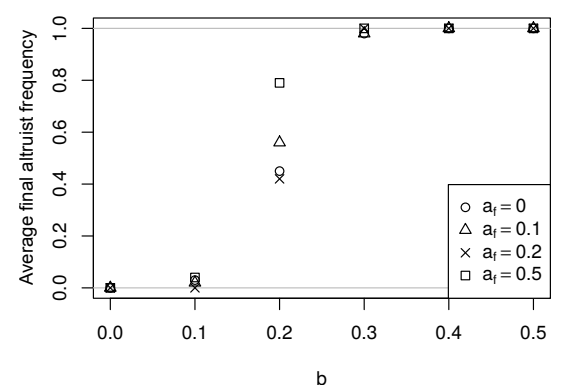

Figure 2: Average final altruist frequency across 100 runs. See Section 3.1 for details.

between different settings of $\kappa_{H}$ (all Bonferroni-Holm corrected p-values above 0.39).

The effect of random fluctuations in host carrying capacity was studied in simulation series $a_{f}$. Results are shown in Figure $2 \mathrm{~d}$. For $b=0$, all simulations led to extinction of altruists, while for $b=0.4$ and $b=0.5$, all simulations showed fixation of altruists. There was a significant effect of the amount of fluctuation, $a_{f}$, for $b=0.2$ (Bonferroni-Holm corrected p-value: $5.6 \cdot 10^{-7}$ ). For $b=0.1$ and $b=0.3$, the effect of $a_{f}$ was not significant (corrected p-value of 0.52 for both).

\subsection{Large populations}

Several simulation series were performed to check how well the condition derived in the theoretical analysis could predict the outcome in a setting of large populations living in finitely many demes. The parameters of all simulation series are shown in Table 2. First, simulation series $\varepsilon$ was performed, to find a suitable cutoff value to account for the discretization (see $\mathrm{C}$ for details), resulting in $\varepsilon=10^{-7}$.

The value $\varepsilon=10^{-7}$ was then used in simulation series $U, N, 2 D$, and $T$, with 100 runs per parameter set. Mean values with standard errors from these simulation series are shown in Figure 3, and root mean squared errors of the simulation results compared to the theoretical prediction are shown in D in Figure 2. 
u

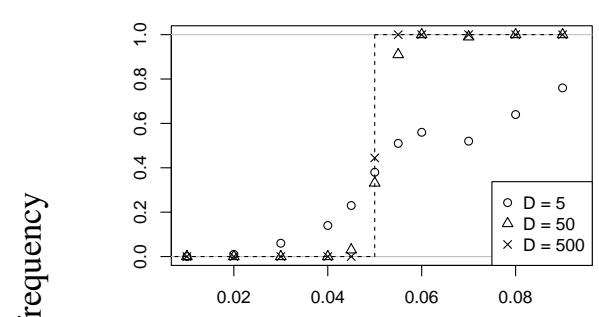

2D

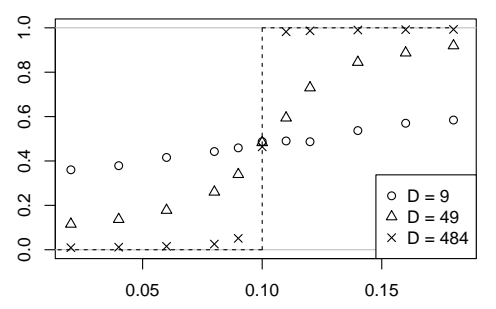

$\mathbf{N}$

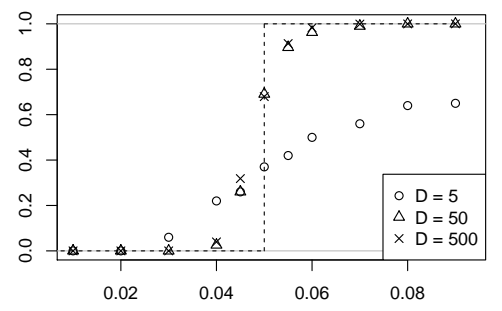

$\mathbf{T}$

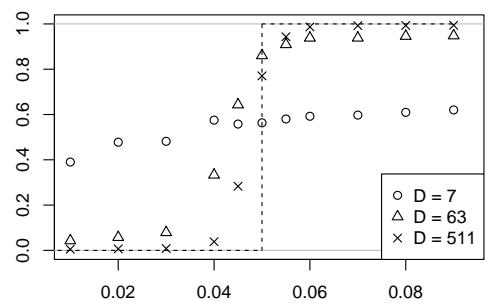

$\beta$

Figure 3: Mean values over 100 simulation runs with uniform migration (U), nearestneighbor migration $(\mathrm{N})$, two-dimensional nearest-neighbor migration (2D), and migration along edges of a binary tree $(\mathrm{T})$. Dashed lines indicate the theoretical predictions.

In simulation series $U$ we checked the prediction from the theoretical results for uniform migration between the demes, with different total deme numbers. For each parameter setting, 100 simulation runs were performed. For $\beta=\alpha$, there were 84 runs with $D=500$ and one run with $D=50$ that did not show extinction or fixation of altruists (Figure 3, top left). All other runs had a final altruist frequency of either zero or one (see Figure 3 in online appendix D for details).

In simulation series $N$ we simulated the process $X$ under nearest-neighbor migration on a circle. For each parameter setting, 100 simulation runs were performed. For $D=50,125$ of the 500 runs with $0.04 \leq \beta \leq 0.06$ showed coexistence of altruists and cheaters, while for $D=500$, coexistence was observed in 425 of the 700 runs with $0.04 \leq \beta \leq 0.08$. All other runs of the simulation series showed fixation or extinction of altruists (Figure 3, top right). For $\alpha=\beta$, a final altruist frequency of either zero or one is observed in 100, 45, and 0 runs (out of a total of 100 runs) for $D=5, D=50$, and $D=500$, respectively (See Figure 4 in online D).

In simulation series $2 D$ we assumed nearest-neighbor migration in two dimensions, i.e. each deme sent migrants to its four immediate neighbors (using a torus structure to avoid boundary effects). For each parameter setting, 100 simulation runs were performed. In none of the simulations, fixation or extinction of altruists occurred, but with 
$D=22^{2}=484$, the final altruist frequency was always close to 0 if $\beta / \alpha<0.8$ and close to 1 if $\beta / \alpha>1.1$ (Figure 3, bottom left; for more details see Figure 5 of online D).

We simulated the process $X$ with migration along edges of a binary tree in simulation series $T$. Demes were represented as nodes of a binary tree and each node exchanged migrants with its parent and its two children. The root sent migrants only to its two children. Neighboring leaves also exchanged migrants. For each parameter setting, 100 simulation runs were performed. In all simulations, the final altruist frequency was strictly between 0 and 1 , but for $D=511$ very close to 0 if $\beta / \alpha<0.04 / 0.05=0.8$ and very close to 1 if $\beta / \alpha>0.06 / 0.05=1.2$ (Figure 3 , bottom right; for more details see Figure 6 in online D).

\section{Discussion}

In Hutzenthaler et al. (2015) we found a condition to determine the success of an altruistic defense allele for deme-structured populations in the limit of large populations in many demes under a complete separation of time scales. In this scenario, the altruistic allele is shown to become fixed in the population if $\alpha<\beta$, it goes extinct if $\alpha>\beta$, and there is coexistence of altruists and cheaters if $\alpha=\beta$. The parameter $\alpha$ denotes the selective disadvantage of altruists (see Section 2.1). The term $\beta$ is defined as

$$
\beta=\rho \frac{\delta}{\delta \nu+\lambda \gamma} \beta_{H},
$$

which can be interpreted as the benefit of defense, scaled with the relative parasite pressure and the amount of randomness in host reproduction. The factor $\frac{\delta}{\delta \nu+\lambda \gamma}$ denotes the potential harm that hosts suffer from parasites. This represents the selective pressure on the host population to evolve defense mechanisms. If the pressure is strong, then the potential benefit of defending is high. In addition, randomness in host reproduction directly benefits the evolution of the altruistic defense allele. This is due to the fact that variation in population sizes between demes enable the Yule-Simpson effect (Yule, 1903; Simpson, 1951; Blyth, 1972). Demes with a high altruist frequency are better protected against parasites and thus have a higher host density. Thereby, they are able to send off more migrants than demes with few altruists. Thus, even though being individually disfavored in each deme, altruists can have an advantage over cheaters in the total population.

\subsection{Small populations}

To obtain the theoretical prediction for fixation or extinction of altruists, Hutzenthaler et al. (2015) assumed infinite population sizes, infinitely many demes, and an infinite time horizon together with a complete separation of ecological and evolutionary time scales (see Figure 1). The aim of this study is to analyze the model under relaxed assumptions in a finite setting. For small population sizes (Section 2.1), the prediction is qualitatively fulfilled, i.e., there is either extinction or fixation of altruists, with a narrow transition range between the two outcomes. However, the value for which this transition occurs does not coincide with the predicted one. The effect can clearly be 
observed in simulation series $\alpha$. Even without altruism providing any benefit-i.e., $b=0$, with $b=\rho \delta /(\delta \nu+\lambda \gamma)=\beta / \beta_{H}$ - the selective disadvantage of altruism needs to be relatively high in order for altruist extinction to be a stable result across simulations (Figure $2 \mathrm{a}$ ). For $b>0$, the inherent randomness in the finite system favors altruism by increasing between-deme variances. This is highlighted by simulation series $\beta_{H}$, which evaluates the process for different values of the amount of randomness in host reproduction, $\beta_{H}$ (Figure $2 \mathrm{~b}$ ). Even for $\beta_{H}=0$, altruism can be favored when $b$ is sufficiently large. In the asymptotic analysis of Hutzenthaler et al. (2015), all randomness is captured in $\beta_{H}$ and altruists always go extinct when $\beta_{H}=0$. Nevertheless, the qualitative influence of $\beta_{H}$ in the simulations behaves according to the theoretical prediction, i.e., increasing $\beta_{H}$ leads to a stronger benefit of altruists (Figure $2 b$ ).

The asymptotic result predicts that there is no influence of the host migration rate $\kappa_{H}$ on the evolution of altruistic defense (as long as $\kappa_{H}>0$ ). In the context of small population sizes, migration rates may play a more important role than for the setting of large populations. Ecological and evolutionary time scales are not fully separated in the finite setting and thus, a large value of $\kappa_{H}$ may mean that migration events interfere with host-parasite interactions. On the other hand, a small value may result in very little mixing of the population and thereby diminish a possible benefit of altruists. However, no significant effect of the host migration rate could be detected in simulation series $\kappa_{H}$ (Figure 2c). Consequently, the amount of gene flow under which the evolution of altruism is favored may not be as limited as suggested by other approaches (Maynard Smith, 1964; Wade, 1978; Berngruber et al., 2013).

The positive effect of random differences in population sizes between demes on the success of altruists is also visible in simulation series $a_{f}$. Random fluctuations in the carrying capacities of the host and parasite populations were added in different amounts. This amplifies between-deme variances and may lead to a more pronounced Yule-Simpson effect. Indeed, larger fluctuations are beneficial for altruism (Figure 2d).

In all simulation series, fixation or extinction of altruists is very stable across different simulation runs with the same parameter settings, except for the narrow transition ranges between the two outcomes. Thus, in line with the theoretical prediction, there is a clear advantage of either altruists or cheaters that can overcome the randomness that may lead to fixation of a disfavored type in the finite setting.

\subsection{Effect of population structure}

Simulations assuming infinite population sizes were performed in order to compare the success of altruists in finite and infinite settings and to investigate effects of population structure. We simulated the process $X$ given by equation (1). It describes the relative frequencies of altruists in the limit of large population sizes under a complete separation of time scales.

The simulations with uniform migration among 500 demes fit the theoretical prediction very well (Figure 3 ). The analytical result could only be obtained in Hutzenthaler et al. (2015) when uniform migration is assumed, allowing for a mean field approximation. Nevertheless, for the other migration schemes investigated, the prediction was also closely met, when the number of demes was large enough. For these other migration schemes, fixation or extinction of altruists was often not reached within 
the given time frame when $\beta$ was very close to $\alpha$. This may be explained by the fact that under uniform migration, population mixing is strongest and any given deme can best be reached by individuals. For simulation series $2 D$ and simulation series $T$, the final altruist frequency stayed strictly within the boundaries of zero and one for all simulations runs across all parameter settings (contrary to simulation series $U$ and simulation series $N$ ). We conclude that, although the specifics of the population structure do not appear to change which type is favored, they can strongly influence the outcome of the system and lead to prolonged times of coexistence. Thus, the specific spatial structure of a natural population needs to be considered when applying theoretical insights. Across all simulations of $X$, the altruistic defense trait could only go to fixation (within the simulated time frame of $2000 \cdot N$ generations) if a large number of demes was assumed.

\subsection{Feedback effects}

As Sibly and Curnow (2011) and Berngruber et al. (2013) point out, the success of an altruistic defense trait depends on the presence of parasites. Thus, benefits of altruistic acts are not constant in the scenario of defense. These fluctuations of effect strengths as well as changing values of relatedness do not need to be considered using the present result. Notably, the current state of host and parasite populations does not enter in the condition for the success of altruism. Instead, the advantage or disadvantage of the defense trait remains fixed over time. Indeed, in most of the simulations, no such negative feedback can be detected and altruists typically reach fixation or extinction. The only exception - for which a negative feedback effect occurs-is simulation series $\beta_{H}$ (Figure 2b). The strong defense of altruists for $b=0.7$ leads to extinction of parasites in most cases (Section 3.1). If this extinction of parasites occurs before the fixation of altruists, then altruism provides no further benefit and cheaters can take over the population. Thus, the benefit of defense can be detrimental to the evolution of altruism, when parasites go extinct in this finite system. This result is similar to the findings of Duncan et al. (2011), who have shown for Paramecium Caudatum that after removing contact to a parasite, costly resistance is no longer maintained and productivity of the host population slowly increases.

\subsection{Comparison to results obtained with other modeling approaches}

Uyenoyama (1979) investigated the evolution of altruism in an island model, assuming a stage of random mixing of the whole population followed by recolonization of islands in each generation. This model goes back to Levene (1953) and has also been utilized by Gillespie (1974). The simplification of assuming a stage of random mixing allows to immediately obtain a one-dimensional diffusion approximation. Uyenoyama (1979) found that for group selection to act, it is not necessary to invoke a mechanism for complete extinction of whole groups. Instead, it is enough if there is maintained variation between the groups, e.g., due to genetic drift or fluctuating environments. Similarly, in our model variation between demes is maintained solely via genetic drift, which is a sufficient force even in large populations, when the processes are analyzed on a large enough time scale. Thus, if some of the other mechanisms to increase and maintain 
genetic variability (such as mutation, as discussed by Lande, 1976) are introduced in the model, this might provide an even larger benefit for altruism.

Slatkin and Wade (1978) show several mechanisms that are advantageous for the evolution of altruism. Among them are small population sizes per deme, extinction of demes, mutation, and migration in groups with small founding population sizes. None of these are satisfied in the present study, yet, genetic drift produces enough variation between demes in order for deme-level selection to counteract the cost of altruism.

Neither the asymptotic analysis of Hutzenthaler et al. (2015) nor our present analysis required the explicit calculation of relatedness values, but it is of course possible to interpret our results from the perspective of inclusive fitness theory. The between-deme variation in the frequency of the defense allele in our model comes from genetic drift and thus from the within-deme relatedness structure. Thus, from the perspective of the causal interpretation of kin selection (Gardner, 2015; Okasha and Martens, 2016) altruists defend their deme because their relatives are overrepresented in the deme. High amounts of deme-level relatedness also imply, however, that relatives compete for limited space or resources. This competition has been shown to potentially cancel the beneficial effects of cooperation and thus prevent the evolution of altruism (Wilson et al., 1992; Taylor, 1992). However, Alizon and Taylor (2008) show how this effect of competition can be reduced by allowing for empty sites in local populations and varying dispersal rates depending on the size of a deme. Thereby, migration increases with increasing density in a deme and competition can be reduced. We observed a similar effect in the present approach, where larger demes send off more migrants and kin competition can be overcome. However, in our scenario, this effect is obtained with constant migration rates, whereas migration rates are either zero or one (depending on the local population size) in the approach of Alizon and Taylor (2008). Platt and Bever (2009) review the mechanisms that reduce effects of kin competition and allow for a spread of altruism in structured populations. Apart from allowing for empty sites, an important concept is that of population elasticity. By allowing local carrying capacities to increase under a high density of cooperators, the impact of kin competition can be weakened. In the present model, kin competition is reduced due to population elasticity. Here, a high altruist frequency reduces parasite pressure and thereby increases the potential local host population size.

Similar to game theoretic approaches such as the Prisoner's dilemma (Axelrod and Hamilton, 1981), cheating appears to be the favorable strategy in the present analysis. Independent of the types of other individuals in a deme, a focal individual has an increased immediate reproductive success by cheating compared to cooperating. Nevertheless, the results show that, in fact, altruism will prevail under suitable conditions. Several explanations have been given for such seemingly contradicting results using the methods of game theory. Including spatial structure in evolutionary games can lead to the evolution of altruism (e.g., Ohtsuki et al., 2006; Fletcher and Zwick, 2007). This, of course, is reflected in the present analysis, which also relies on spatial structure and differences among demes to achieve an advantage of altruists. Furthermore, introducing repeated interactions has been shown to increase the long-term success of cooperators compared to cheaters when considering the Prisoner's dilemma (Trivers, 1971; Nowak, 2006). In the present modeling approach, this insight may be applied as well. There are not merely single interactions between individuals, but instead partners 
will stay together (and interact) for considerable amounts of time. This is achieved by a relatively weak migration process, such that individuals do not change demes too quickly.

Débarre et al. (2012) investigated the evolution of altruistic defense traits, in particular suicide upon infection and reduction of transmission, in a host-parasite system with spatial structure modeled as a triangular lattice. They concluded that suicide upon infection with an additional fecundity cost for the ability to detect the parasite can evolve in a structured population if the parasite is sufficiently harmful to the host population. This is in line with the definition of $\beta$ in the present model that captures the parasite-induced selection pressure on hosts as well as the necessity of $\kappa_{H}$ being positive, which ensures the population structure in the present model.

\subsection{Future directions}

It might be insightful to extend the present modeling approaches to allow for a wider spectrum of defense strategies depending on several loci. This may lead to more pronounced differences between demes and thus, provide better conditions for altruism. Furthermore, considering a polygenic trait with mutation may lead to increased variation between demes and thus also favor altruists (Lande, 1976). The model discussed here does not allow for coevolutionary dynamics, but some conclusions can be drawn. Counter-adaptations of parasites to the defense strategies of altruists become relevant only after altruists have reached a high frequency. Since the present results predict that there is no coexistence of different host strategies, i.e., altruism and cheating, it is likely that fixation of altruism will occur shortly after parasites feel the pressure of evolving counter-adaptations. This may result in a step-wise arms race, allowing the present results to be applied successively. Nevertheless, explicit modeling of coevolutionary dynamics may provide further insights, because parasite virulence can be highly dependent on evolutionary dynamics of the host, as shown by Best et al. (2009).

\section{References}

\section{References}

Abbot, P., Abe, J., Alcock, J., Alizon, S., Alpedrinha, J. A., Andersson, M., Andre, J.-B., van Baalen, M., Balloux, F., Balshine, S., et al. (2011). Inclusive fitness theory and eusociality. Nature, 471(7339):E1-E4.

Alizon, S. and Taylor, P. (2008). Empty sites can promote altruistic behavior. Evolution, 62(6):1335-1344.

Antonovics, J. and Thrall, P. H. (1994). The cost of resistance and the maintenance of genetic polymorphism in host-pathogen systems. Proceedings of the Royal Society of London B: Biological Sciences, 257(1349):105-110.

Axelrod, R. and Hamilton, W. D. (1981). The evolution of cooperation. Science, 211(4489):1390-1396. 
Berngruber, T. W., Lion, S., and Gandon, S. (2013). Evolution of suicide as a defence strategy against pathogens in a spatially structured environment. Ecol. Lett., $16(4): 446-453$.

Best, A., Webb, S., White, A., and Boots, M. (2011). Host resistance and coevolution in spatially structured populations. Proc. R. Soc. B, 278(1715):2216-2222.

Best, A., White, A., and Boots, M. (2009). The implications of coevolutionary dynamics to host-parasite interactions. Am. Nat., 173(6):779-791.

Bijma, P., Muir, W. M., and Van Arendonk, J. A. (2007). Multilevel selection 1: Quantitative genetics of inheritance and response to selection. Genetics, 175(1):277-288.

Birch, J. and Okasha, S. (2015). Kin selection and its critics. BioScience, 65(1):22-32.

Blyth, C. R. (1972). On Simpson's paradox and the sure-thing principle. J. Am. Stat. Assoc., 67(338):364-366.

Boots, M., Best, A., Miller, M. R., and White, A. (2009). The role of ecological feedbacks in the evolution of host defence: what does theory tell us? Phil. Trans. R. Soc. B, 364(1513):27-36.

Bowers, R. G., Boots, M., and Begon, M. (1994). Life-history trade-offs and the evolution of pathogen resistance: Competition between host strains. Proceedings of the Royal Society of London B: Biological Sciences, 257(1350):247-253.

Brown, D. H. and Hastings, A. (2003). Resistance may be futile: dispersal scales and selection for disease resistance in competing plants. J Theor Biol, 222(3):373-88.

Cotter, S. C., Kruuk, L. E., and Wilson, K. (2004). Costs of resistance: genetic correlations and potential trade-offs in an insect immune system. J Evol Biol, 17(2):421-9.

Débarre, F., Lion, S., van Baalen, M., and Gandon, S. (2012). Evolution of host lifehistory traits in a spatially structured host-parasite system. Am. Nat., 179(1):52-63.

Diggle, S. P., Griffin, A. S., Campbell, G. S., and West, S. A. (2007). Cooperation and conflict in quorum-sensing bacterial populations. Nature, 450(7168):411-414.

Duncan, A. B., Fellous, S., and Kaltz, O. (2011). Reverse evolution: selection against costly resitance in disease-free microcosm populations of Paramecium Caudatum. Evolution, 65(12):3462-3474.

Fletcher, J. A. and Zwick, M. (2007). The evolution of altruism: game theory in multilevel selection and inclusive fitness. J. Theor. Biol., 245(1):26-36.

Frank, S. A. (1998). Inducible defence and the social evolution of herd immunity. Proc Biol Sci, 265(1408):1911-3.

Gardner, A. (2015). The genetical theory of multilevel selection. J. Evol. Biol., 28(2):305-319. 
Gillespie, J. (1974). Polymorphism in patchy environments. Am. Nat., 108(960):145151.

Hamilton, W. D. (1963). The evolution of altruistic behavior. Am. Nat., 97:354-356.

Hamilton, W. D. (1964a). The genetical evolution of social behaviour. I. J. Theor. Biol., $7(1): 1-16$.

Hamilton, W. D. (1964b). The genetical evolution of social behaviour. II. J. Theor. Biol., 7(1):17-52.

Hölldobler, B. and Wilson, E. O. (2009). The superorganism: the beauty, elegance, and strangeness of insect societies. WW Norton \& Company, New York.

Hutzenthaler, M., Jordan, F., and Metzler, D. (2015). Altruistic defense traits in structured populations. arXiv:1505.02154v1.

Lande, R. (1976). The maintenance of genetic variability by mutation in a polygenic character with linked loci. Genet. Res., 26(3):221-235.

Levene, H. (1953). Genetic equilibrium when more than one ecological niche is available. Am. Nat., pages 331-333.

Maynard Smith, J. (1964). Group selection and kin selection. Nature, 201:1145-1147.

Metzler, D., Jordan, F., Pamminger, T., and Foitzik, S. (2016). The influence of space and time on the evolution of altruistic defence: the case of ant slave rebellion. $J$ Evol Biol, 29(5):874-86.

Nowak, M. A. (2006). Five rules for the evolution of cooperation. Science, 314(5805):1560-1563.

Nowak, M. A. and May, R. M. (1992). Evolutionary games and spatial chaos. Nature, 359(6398):826-829.

Nowak, M. A., Tarnita, C. E., and Wilson, E. O. (2010). The evolution of eusociality. Nature, 466(7310):1057-1062.

Ohtsuki, H., Hauert, C., Lieberman, E., and Nowak, M. A. (2006). A simple rule for the evolution of cooperation on graphs and social networks. Nature, 441(7092):502505 .

Okasha, S. and Martens, J. (2016). The causal meaning of Hamilton's rule. R Soc Open Sci, 3(3):160037.

Pamminger, T., Foitzik, S., Metzler, D., and Pennings, P. S. (2014). Oh sister, where art thou? Spatial population structure and the evolution of an altruistic defence trait. J. Evol. Biol., 27(11):2443-2456.

Platt, T. G. and Bever, J. D. (2009). Kin competition and the evolution of cooperation. Trends Ecol. Evol., 24(7):370-377. 
Price, G. R. (1970). Selection and covariance. Nature, 227:520-21.

Queller, D. C. (1992). Quantitative genetics, inclusive fitness, and group selection. American Naturalist, 139(3):540-558.

R Core Team (2015). R: a language and environment for statistical computing. $\mathrm{R}$ Foundation for Statistical Computing, Vienna, Austria.

Rousset, F. and Lion, S. (2011). Much ado about nothing: Nowak et al.'s charge against inclusive fitness theory. J. Evol. Biol., 24(6):1386-1392.

Schliekelman, P. (2007). Kin selection and evolution of infectious disease resistance. Evolution, 61(6):1277-88.

Sibly, R. M. and Curnow, R. (2011). Selfishness and altruism can coexist when help is subject to diminishing returns. Heredity, 107(2):167-173.

Simpson, E. H. (1951). The interpretation of interaction in contingency tables. $J . R$. Stat. Soc. B, 13(2):238-241.

Siva-Jothy, M. T., Moret, Y., and Rolff, J. (2005). Insect Immunity: An Evolutionary Ecology Perspective. Advances in Insect Physiology, 32:1-48.

Slatkin, M. and Wade, M. (1978). Group selection on a quantitative character. Proc. Nat. Acad. Sci. USA, 75(7):3531-3534.

Sober, E. and Wilson, D. S. (1998). Unto others: The evolution and psychology of unselfish behavior. Harvard University Press, Cambridge, MA.

Taylor, P. (1992). Altruism in viscous populations-an inclusive fitness model. Evol. Ecol., 6(4):352-356.

Traulsen, A. and Nowak, M. A. (2006). Evolution of cooperation by multilevel selection. Proc. Natl. Acad. Sci. USA, 103(29):10952-10955.

Trivers, R. L. (1971). The evolution of reciprocal altruism. Q. Rev. Biol., 46(1):35-57.

Uyenoyama, M. K. (1979). Evolution of altruism under group selection in large and small populations in fluctuating environments. Theor. Popul. Biol., 15(1):58-85.

Van Veelen, M., García, J., Sabelis, M. W., and Egas, M. (2012). Group selection and inclusive fitness are not equivalent; the Price equation vs. models and statistics. $J$. Theor. Biol., 299(0):64-80.

Wade, M. J. (1978). A critical review of the models of group selection. Q. Rev. Biol., 53(2):101-114.

Wade, M. J. (1982). Evolution of interference competition by individual, family, and group selection. Proc. Natl. Acad. Sci. USA, 79(11):3575-3578. 
West, S. A., Griffin, A. S., and Gardner, A. (2007). Social semantics: altruism, cooperation, mutualism, strong reciprocity and group selection. J. Evol. Biol., 20(2):415432 .

Wilkinson, G. S. (1984). Reciprocal food sharing in the vampire bat. Nature, 308(5955):181-184.

Wilkinson, G. S. (1990). Food sharing in vampire bats. Sci. Am., 262(2):76-82.

Williams, P., Winzer, K., Chan, W. C., and Camara, M. (2007). Look who's talking: communication and quorum sensing in the bacterial world. Phil. Trans. R. Soc. B, 362(1483):1119-1134.

Wilson, D. S., Pollock, G., and Dugatkin, L. A. (1992). Can altruism evolve in purely viscous populations? Evol. Ecol., 6(4):331-341.

Wynne-Edwards, V. C. (1963). Intergroup selection on the evoliution of social systems. Nature, 200:623-626.

Yule, G. U. (1903). Notes on the theory of association of attributes in statistics. Biometrika, 2(2):121-134. 